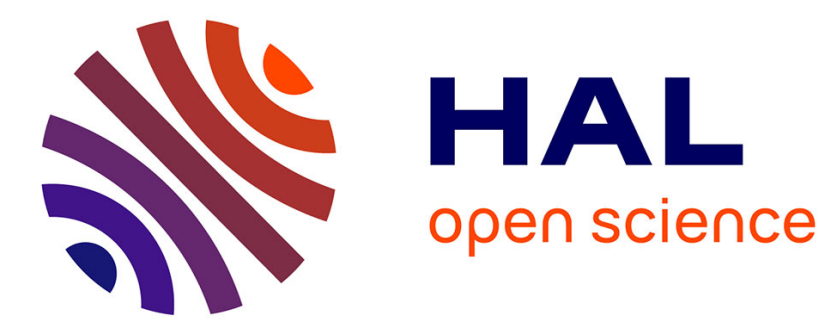

\title{
Dissolution des liquides et des solides dans les gaz
}

P. Villard

\section{To cite this version:}

P. Villard. Dissolution des liquides et des solides dans les gaz. J. Phys. Theor. Appl., 1896, 5 (1), pp.453-461. 10.1051/jphystap:018960050045301 . jpa-00239939

\section{HAL Id: jpa-00239939 https://hal.science/jpa-00239939}

Submitted on 1 Jan 1896

HAL is a multi-disciplinary open access archive for the deposit and dissemination of scientific research documents, whether they are published or not. The documents may come from teaching and research institutions in France or abroad, or from public or private research centers.
L'archive ouverte pluridisciplinaire HAL, est destinée au dépôt et à la diffusion de documents scientifiques de niveau recherche, publiés ou non, émanant des établissements d'enseignement et de recherche français ou étrangers, des laboratoires publics ou privés. 


\section{DISSOLUTION DES LIQUIDES ET DES SOLIDES DANS LES GAZ ;}

Par M. P. VILLARD.

En 1880, MM. Hannay et Hogarth (') réussissaient à dissoudre des corps solides, tels que le bromure et l'iodure de potassium, dans l'alcool gazeux chauffé au-delà de sa température critique. La même année, M. Cailletet $\left({ }^{2}\right)$ dissolvait l'anhydride carbonique liquide dans de l'air comprimé. Ce ne sont pas là les seuls essais faits dans cette voie, mais personne ne parait avoir cherché si, à la température ordinaire, les gaz possèdent la propriété de dissoudre des corps liquides ou solides dans les circonstances ordinaires. Une telle propriété existe cependant, et il est facile de la mettre en évidence. Un corps solide, par exemple, plongé dans un espace rempli de gaz, peut disparaître en tant qu'objet solide, se diffuser dans la masse gazeuse, et cela en quantité parfois bien supérieure à celle qui suffirait à saturer le même espace supposé vide de gaz. On est fondé à dire que le corps considéré a pris l'ètat physique du gaz auquel il s'est mélangé, c'est-à-dire l'état gazeux : on est en présence d'une dissolution gazeuse. Pour simplifier le langage, on peut considérer comme faisant partie de la dissolution la vapeur saturée normale telle qu'elle se serait produite en l'absence de tout dissolvant.

(') Proceed. of the Roy. Soc. of London, t. XXX, p. 178. - Journ. de Phys., $1^{\text {ro }}$ série, t. IX, p. $415 ; 1880$.

() Journ. de Phys, $1^{\text {re }}$ série, t. IX, p. $193 ; 1880$. 
Voici maintenant les résultats obtenus avec divers gaz, et, sauf indication contraire, à la température ordinaire ( $+17^{\circ}$ environ).

Oxygène. - Air. - Hydrogène. - Il est facile d'observer la dissolution, dans l'oxygène, d'un liquide tel que le brome, dont la vapeur est colorée. Il suffit de mettre une goutte de brome à la partie inférieure d'un tube étroit en cristal, et de faire arriver dans ce tube, par la partie supérieure, de l'oxygène comprimé, jusqu'à atteindre 200 atmosphères par exemple. La vapeur de brome est d'abord refoulée, et se rassemble près du liquide; mais en même temps sa coloration devient plus intense. Inclinant le tube, cette couche de vapeur s'étale et se diffuse, de nouvelles vapeurs se forment au contact du liquide, et bientôt l'atmosphère du tube prend une teinte beaucoup plus foncée qu'avant la compression. On obtient le même résultat en laissant la diffusion s'opérer d'elle-même, le tube restant immobile. Vient-on à abaisser la pression, la coloration s'affaiblit, et des gouttelettes de liquide se déposent sur les parois; ces gouttelettes disparaissent quand on comprime de nouveau. Il y a donc réellement dissolution, dans l'oxygène, d'une quantité sensible de brome.

Vers 300 atmosphères, la coloration obtenue dépasse de beaucoup celle de l'eau bromée; elle est sensiblement la même qu'avec une épaisseur au moins six fois plus grande de vapeur de brome saturée dans le vide.

Il n'est, d'ailleurs; pas nécessaire d'atteindre des pressions aussi élevées pour observer le phénomène. L'effet produit par l'oxygène est déjà appréciable, par comparaison, à partir de 4 atmosphères; il devient très marqué si l'on atteint 50 atmosphères ou 100 atmosphères. Sous cette dernière pression, la densité du brome dissous, à en juger par sa teinte, paraît être environ triple de la densité de la vapeur de brome saturée ordinaire.

L'emploi de tubes scellés de dimensions convenables permet de comparer facilement les résultats obtenus sous diverses pressions, jusqu'au-delà de 300 atmosphères.

L'air se comporte à peu près comme l'oxygène : cependant, toutes choses égales d'ailleurs, la coloration obtenue est un peu plus faible dans l'air que dans l'oxygène.

L'iode se dissout également, en quantité sensible, dans l'oxygène, mais le phénomène n'est bien net que si l'on atteint une centaine d'atmosphères, et dans des tubes de 5 millimètres à 6 millimètres au moins de diamètre intérieur. 
L'hydrogène ne possède qu'un très faible pouvoir dissolvant, que l'on peut cependant mettre en évidence avec le brome, sous des pressions de 200 atmosphères à 300 almosphères.

Formène. - Le formène convient très bien pour dissoudre, en quantités notables, des liquides tels que le chlorure d'éthyle, le sulfure de carbone, l'alcool, ou des solides, comme le camphre ou la paraffine. Avec ce dissolvant, il n'est pas nécessaire d'atteindre de fortes pressions pour constater que la solubilité d'un liquide ou d'un solide dans un gaz peut n'ètre pas très faible, de l'ordre du millième ou du centième par exemple. Ainsi le chlorure d'éthyle peut se dissoudre dans cinq ou six fois son volume de formène comprimé à moins de 200 atmosphères. $\Lambda 200$ atmosphères vers $+17^{\circ}$, la quantité de chlorure d'éthyle que l'on peut dissoudre devient telle que, si on cherche à la dépasser, c'est au contraire le gaz qui se dissout en totalité dans le liquide. Les deux fluides sont réellement devenus miscibles l'un avec l'autre, car si on a soin de ne pas les agiter pendant la compression, toute surface de séparation du liquide et du gaz disparaît à 200 atmosphères. Le même phénomène se produit avec le sulfure de carbone sous une pression de ə̆ŏ0 atmosphères environ à la température ordinaire, et de 2000 atmosphères à $+130^{\circ}$.

L'iode se dissout facilement dans le formène, et lui communique une coloration violette qui devient intense à 300 atmosphères, même sous une épaisseur de 2 millimètres. On peut également dissoudre en quantités visibles du camphre ou de la paraffine. Par décompression les corps dissous se déposent : le camphre cristallise sur les parois des tubes, la paraffine se précipite en paillettes brillantes.

$\dot{E}$ thylène. - L'iode se dissout dans l'éthylène en quantité suffisante pour le colorer en violet foncé sous une épaisseur de 2 millimètres vers 300 atmosphères. Il se forme ensuite, au bout d'une heure ou deux, de l'iodure d'éthylène dont la majeure partie reste en dissolution. En abaissant la pression, l'iodure cristallise.

Au-dessus de 150 atmosphères, l'éthylène dissout abondamment la paraffine; par décompression celle-ci reprend l'état solide et forme dans le tube un véritable feutrage.

L'acide stéarique ordinaire (bougie stéarique) se dissout également, mais moins facilement que la paraffine.

Le camphre donne lieu à un phénomène particulier : si la pression est faible, il prend directement l'état gazeux ; vers 150 atmosphères la partie non dissoute se liquéfie et augmente de volume. Ce liquide 
se dissout ensuite dans l'éthylène si on élève la pression, et devient miscible avec le gaz vers 10 atmosphères. Les mêmes phénomènes se passent en sens inverse quand la pression diminue.

Anhydride carbonique. - Protoxyde d'azole. - L'anhydride carbonique comprimé, à l'état de vapeur saturée ou non, dissout sensiblement l'iode et se colore en violet. Mais le pouvoir dissolvant des gaz liquéfiables ne parait présenter rien de particulier. Ainsi, dans le protoxyde d'azote à 20 atmosphères, vers $+17^{\circ}$, le brome se dissout comme dans l'oxygène à 40 atmosphères environ.

\section{PhÉNomènes CRITIQUes.}

Si l'on met, par exemple, du chlorure d'éthyle en présence de formène comprimé, on constate que pour une pression déterminée, 200 atmosphères environ à la température ordinaire, le niveau du liquide se transforme en une zone de transition. Par agitation, les deux fluides forment alors un mélange homogène. On est en présence d'un véritable point critique, déjà observé par M. Cailletet (loc. cit.) avec l'anhydride carbonique et l'air. La pression pour laquelle le ménisque disparaît est parfaitement définie pour une température donnée et peut être mesurée avec précision ; nous l'appellerons pression crilique.

Les phénomènes que l'on observe au voisinage de ce point critique particulier sont tout à fait semblables à ceux que présentent les tubes Natterer ordinaires, et, à certains égards, beaucoup plus marqués. La description en sera facile en définissant les conditions de l'expérience de la manière suivante :

Dans un tube de volume invariable, maintenu à température constante, introduisons successivement des quantités de plus en plus grandes de chlorure d'éthyle par exemple, puis ajoutons la quantité de formène nécessaire pour obtenir la pression voulue. Voici alors ce qu'on observe, le tube restant immobile :

$\mathbf{1}^{\circ}$ Le niveau du liquide reste sensiblement fixe pendant que la pression s'élève ;

$2^{\circ}$ Quand on atteint la pression critique, le ménisque disparaît où il se trouve, et sa transformation en une zone de transition peut s'observer avec des tubes très diversement remplis : à cet égard la latitude est plus grande que dans les tubes Natterer; 
$3^{\circ}$ La zone de transition persiste pendant un temps considérable, même si on dépasse de beaucoup la pression critique. Il faudrait attendre des journées entières pour obtenir l'homogénéité du contenu du tube. Ce phénomène est également beaucoup plus marqué que dans les tubes Natterer remplis avec un gaz pur.

Les choses se passent tout autrement si l'on agite :

$1^{\circ}$ Le niveau du liquide se déplace notablement pendant la conpression, et atteint généralement l'une ou l'autre extrémité du tuke avant qu'on n'ait réalisé la pression critique.

$2^{\circ}$ Le niveau ne peut être observé jusqu'au point critique que pour un remplissage déterminé. Il y a donc un volume critique pour le liquide considéré, dans le dissolvant choisi, et à une température donnée. Quand on approche de la pression critique, les indices des deux fluides deviennent manifestement très voisins et le ménisque de séparation est à peine visible; au moment où ce ménisque disparaît, la transition entre les deux fluides est beaucoup moins marquée que précédemment.

$3^{\circ}$ Quand le ménisque disparaît, l'homogénéité s'obtient en quelques secondes par agitation. Si on décomprime à ce moment, on obtient une condensation uniforme.

Dans le cas des tubes Natterer ordinaires, ces divers phénomènes s'expliquent aisément, comme je l'ai montré (1), par un défaut d'équilibre thermique. Ici la cause vaporisatrice n'est pas l'élévation de la température, mais l'action du dissolvant. C'est à un défaut d'équilibre entre celui-ci et le liquide que nous devons attribuer les phénomènes en question.

Si nous avons soin, pendant que la pression s'élève, d'agiter le liquide en présence du dissolvant gazeux, la saturation réciproque des deux fluides sera à chaque instant assurée; ce sont là les conditions normales de l'expérience. Le liquide. en se saturant de gaz, augmentera de volume; c'est là un fait général connu el que j’ai constamment observé avec nombre de corps. La densité du liquide, abstraction faite du poids du gaz dissous, ira en diminuant. En même temps le liquide se dissoudra de plus en plus dans le gaz dont la pression augmente, et la densité dè sa vapeur ira croissant. Pour une pression suffisante, ces deux densités seront identiques; on sera au point critique. On peut maintenant répéter les raisonnements faits

(1) Voir p. 2507 de ce volume. 
à propos des gaz liquéfiés, et il est bien évident que, si le poids du liquide en expérience est différent de celui qui permet de réaliser la densité critique dans le tube considéré, le niveau atteindra l'une des extrémités du tube avant le point critique. Autrement dit, sauf pour un remplissage déterminé, tout le liquide se dissoudra dans le gaz avant la pression critique, ou inversement la totalité du gaz se dissoudra dans le liquide. Dans le premier cas le niveau s'abaissera, dans le second il s'élèvera.

Si le remplissage critique est réalisé, vapeur et liquide auront même densitë en arrivant au point critique; on peut considérer comme évident qu'à ce moment le liquide saturé de gaz et le gaz saturé par la vapeur du liquide sont identiques; d'ailleurs, s'il en était autrement, l'égalité des indices n'aurait pas lieu.

Comprimons, au contraire, sans agiter: la diffusion réciproque des fluides en présence ne s'effectuera que très lentement, beaucoup plus lentement que la diffusion de la chaleur dans un tube Natterer ordinaire. La saturation ne sera à chaque instant réalisée qu'au voisinage immédiat du niveau. A quelque distance de celui-ci, les densités différeront beaucoup plus que si l'on agitait. La majeure partie du liquide et du gaz seront à peine intéressés par le phénomène de dissolution, et le niveau changera à peine de place.

En arrivant à la pression critique, les deux couches infiniment minces qui comprennent entre elles le ménisque auront, à un infiniment petit près, la même composition et le même indice. De part et d'autre, dans les deux fluides, la composition variera d'une façon continue, très rapidement dans la région où se trouve le niveau. Dans ces conditions, le ménisque ne peut être visible, il est compris dans une zone où l'indice et la densité varient sans discontinuité. On n'éviterait ce phénomène qu'en faisant varier la pression de quantités infiniment petites et agitant chaque fois l'appareil.

Au-delà du point critique l'homogénéité ne sera obtenue que quand la diffusion sera complètement terminée, c'est-à-dire au bout d'un temps très considérable; il n'en sera évidemment pas de même si on agite.

Les phénomènes qui viennent d'être décrits ne sont pas les seuls qu'on observe (1), et, pour montrer jusqu'à quel point la ressem-

(1) Voir à ce sujet le mémoire posthume de Thomas Andrews : Sur les propriétés de la matière à l'état gazeux et à l'état liquide dans diverses conditions de température et de pression. - (Philos. trans. of the R. Soc. of London, t. CLXXVIII, p. 45; 1887. - Annales de Chimie et de Physique, 6série, t. XIII, p.411.) 
blance est complète avec ce qui se passe dans les tubes Natterer, j'ajouterai que, si on abaisse lentement la pression dans le tube à deux fluides, après l'avoir porté près de la pression critique, il se produit une ébullition et une pluie simultanées, comme dans un tube Natterer que l'on refroidit. Ce phénomène s'explique ici sans difficulté : par décompression le gaz abandonne du liquide, et réciproquement.

Les expériences précédentes peuvent être répétées avec d'autres corps que le formène et le chlorure d'éthyle; les résultats obtenus sont semblables, ce qui montre qu'il s'agit d'un fait général.

Pour deux fluides donnés, la pression critique varie avec la température, et, au moins à partir d'un certain point, décroît quand la température s'élève, pour aboutir à la pression critique ordinaire du liquide seul, tout dissolvant devenant à ce moment inutile.

application a la dissociation.

Considérons, par exemple, un composé solide dissociable, dont la décomposition donne naissance à un solide fixe et à un gaz. Enfermons ce corps dans une enceinte où le vide a été fait, puis élevons la température : la tension de dissociation, qui augmente sans limiles quand la température s'élève, pourra toujours ètre réalisée si le gaz qui se dégage est permanent. Il n'en sera plus de même si le gaz est liquéfiable. A une certaine température $\Theta$, la tension de dissociation, croissant généralement plus vite que la force élastique maxima du gaz, deviendra égale à cette dernière. $\mathrm{Au}$-dessus de $\Theta$, la tension de dissociation ne pourra être atteinte, l'équilibre chimique ne pourra ètre réalisé; il semble que l'existence du composé considéré sera devenue impossible par suite de cette circonstance particulière que le gaz mis en liberté se liquéfiera avant d'avoir atteint une pression suffisante pour limiter la décomposition.

L'intervention d'un gaż inerte fait disparaître cette anomalie; mais, avant de montrer quel rôle ce gaz va jouer, remplaçons la notion de tension de dissociation par celle de densité de dissocialion. Cette expression exprime, aussi bien que la précédente, les résultats de l'expérience, et présente sur elle deux avantages principaux : dans le cas d'un mélange de gaz, on ne peut faire que des hypothèses sur la pression individuelle des composants du mélange; leur densité peut, au contraire, être connue. En second lieu l'expression proposée 
convient également à la dissociation du carbonate de calcium dans le vide et à celle du sulfate mercurique dans l'eau.

Revenons maintenant au cas qui nous occupe, et supposons la température supérieure à $\Theta$. Nous pouvons, au moyen d'un gaz iner te, dissoudre le gaz liquéfié mis en liberté par la chaleur, et lui faire rıprendre l'état gazeux par voie de dissolution, sa densité pourra ainsi devenir très supérieure à sa clensité maxima dans le vide, et la densité de dissociation pourra être atteinte et dépassée, comme si le gaz était permanent. Dans ces conditions l'équilibre chimique sera possible, et le composé en question pourra exister au-delà de la tem. pérature $A$.

Cette théorie s'applique, presque sans modifications, aux hydrates solides dissociables formés par l'eau avec un certain nombre de gaz ou de liquides. La formation et la décomposition de ces corps sont d'une observation particulièrement facile en raison du fait que ces hydrates se distinguent de leurs constituants par leur état physique, au moins au-dessus de $0^{\circ}$. Pour plus de clarté, nous ferons abstraction de la vapeur émise par l'eau, à laquelle on pourrait d'ailleurs appliquer le raisonnement précédent.

Soit, par exemple, l'hydrate de brome: la température $\Theta$ particulière à ce composé est $+6^{\circ}$; si l'on fait intervenir l'air ou l'hydrogène, qui ont la propriété de dissoudre le brome, cette température peut être dépassée sans que l'hydrate se décompose. Dans l'air à la pression ordinaire on atteint déjà $+6^{\circ}, 8$ ou $+7^{\circ}$, sous une pression de 1 0 atmosphères, la formation de l'hydrate peut être observée jusque vers $+20^{\circ}$.

L'hydrogène est beaucoup moins efficace, et ne permet d'atteindı $\mathrm{e}$ $+9^{\circ}$ que sous une pression de 200 atmosphères environ; or on a vu précisément que le brome est moins soluble dans l'hydrogène que dans l'air.

On obtient des résultats analogues avec les hydråtes de chlorure d'éthyle, d'iodure de méthyle, de chlorure de méthylène, de sulfure de carbone, etc.

Remarquons, en terminant, que les phénomènes de dissociation constituent en quelque sorte un réactif très sensible de la dissolution d'un liquide dans un gaz. La moindre variation de la densité d'une vapeur peut en effet se traduire par une rupture de l'équilibre chimique, c'est-à-dire par la formation ou la destruction d'un composé. C'est ainsi que la dissolution du brome dans l'air à la pression ordi- 
BENOIST. - ÉLECTROSCOPE A TROIS FEUILLES 461 naire est absolument inappréciable à la vue; elle suffit cependant pour élever de près de $1^{\circ}$ la température de décomposition, en vase clos, de l'hydrate de brome. 